\title{
Variables psicosociales y salud en mujeres con cáncer de mama
}

\section{Psychosocial variables and health on women with breast cancer}

\author{
Dehisy Marisol Juárez García ${ }^{1}$ y René Landero Hernández \\ Universidad Autónoma de Nuevo León, México
}

(Recepción: Septiembre 2009 - Aceptación: Diciembre 2009)

\begin{abstract}
Resumen
Los objetivos de este trabajo fueron: describir las variables sociodemográficas y los niveles y la relación entre el estrés, el apoyo social, el optimismo y la salud percibida en un grupo de mujeres con cáncer de mama, y determinar los predictores de la salud percibida en un grupo de mujeres con cáncer de mama. Método: se realizó un estudio transversal no experimental con una muestra no probabilística en 72 mujeres diagnosticadas con cáncer de mama. Los Resultados principales encontrados en el grupo de mujeres con cáncer de mama fueron: un nivel moderado de estrés, un nivel alto de apoyo social, optimismo y salud. Se encontraron relaciones significativas entre: estrés y apoyo social, estrés y optimismo, estrés y salud y optimismo y salud. Las variables predictoras de la salud percibida usando regresión múltiple fueron estrés y optimismo, estas variables explican el 22.3\% de la varianza.

Palabras clave: Cáncer de mama, estrés, optimismo, variables psicosociales, salud.
\end{abstract}

\begin{abstract}
The objectives of this study were: 1 ) to describe the socio-demographic variables of a group of women with breast cancer, 2) to describe the levels and the relationship between stress, social support, optimism and health in a group of women with breast cancer and 3) to determine the health predictors in a group of women with breast cancer. Method: A cross sectional study with a non-probabilistic sample with 72 women breast cancer diagnosed. The main results founded in the group of women with breast cancer were: a moderate level of stress, a high level of social support, optimism and health. Significant relationships were found between: stress and social support, stress and optimism, stress and health and optimism and health. The perceived health's predictors, using a multiple regression were stress and optimism; those variables explain the $22.3 \%$ of variance.
\end{abstract}

Key Words: Breast cancer, stress, optimism, psychosocial variables, health.

1 Correspondencia: Dehisy Marisol Juárez García y René Landero Hernández. Posgrado Facultad de Psicología de la Universidad Autónoma de Nuevo León. Mutualismo 110, Col. Mitras Centro, Monterrey, N. L., México. CP 64460 E-mail: dehisy_jg@yahoo.com.mx rene.landerohr@uanl.edu.mx 


\section{Introducción}

El cáncer de mama es la causa principal de muerte en mujeres entre 40 y 50 años de edad en los países desarrollados; en México es la segunda causa de muerte entre las mujeres mayores de 25 años de edad (Secretaria de Salud, 2007). Según Cárdenas y Sandoval (2006) la prevalencia de cáncer de mama en México se ha incrementado en los últimos treinta años, pasando del decimoquinto al segundo lugar general como causa de muerte y ocupando el primer lugar en mujeres de 35 años. Estos datos epidemiológicos hacen del cáncer mama un tema de vital importancia a nivel políticosanitario, sin embargo también es un tema de interés para los profesionales de salud mental (Cervera \& Aubá, 2005), debido a las consecuencias psicológicas y sociales complejas que implica su diagnóstico y tratamiento (Vinaccia \& Orozco, 2005).

El cáncer de mama es una enfermedad muy temida debido a sus características como padecimiento (dolor, recaídas, muerte) y a los efectos secundarios de los tratamientos utilizados para combatirla (Ehrenzweig, 2006).

Por otro lado, la conceptualización de salud ha tomado un enfoque biospsicosocial dejando atrás la percepción como ausencia de enfermedad y definiéndose según la OMS como el completo bienestar físico, psicológico y social.

La interacción entre los factores biológicos, psicológicos y sociales altera la respuesta inmunológica facilitando la aparición de enfermedad (Klinger et al., 2005), se ha encontrado que diferentes comportamientos influyen en las células inmunes aumentando o disminuyendo su actividad, durante periodos prolongados (Pereyra, 2006).

Según Vera-Villarroel y Buela-Casal (1999) cada vez hay más datos en los que se relacionan las variables psicológicas con el curso de la enfermedad de cáncer, sin embargo esta relación no es planteada como causal sino como facilitadora de su aparición, curso o posible remisión, como lo mencionan Arbizu (2000) y Sirera, Sánchez y Camps (2006), la alteración del sistema inmune puede activar el desarrollo o crecimiento de las enfermedades neoplásicas.

Respecto a esto, se he encontrado que las situaciones interpretadas como estresantes pueden generar diferentes sentimientos negativos como miedo, ira y depresión, estas emociones activan mecanismos bioquímicos a nivel del eje hipotalámico-hipofisario-adrenal, que a su vez suprimen la respuesta inmune (Gómez \& Escobar, 2002), además la exposición continua a situaciones estresantes eleva los niveles de cortisol lo que indica la activación de la corteza suprarrenal (Kirschbaum et al., 1995).

Se ha encontrado que las intervenciones cognitivo conductuales realizadas para reducir el estrés en mujeres con cáncer de mama disminuyen los niveles de cortisol en la sangre, mejoran el significado de la experiencia de vivir con cáncer y aumentan la habilidad para relajarse (Cruess et al., 2000; Phillips et al., 2008); además de mejorar diversos aspectos del ajuste psicosocial como la recuperación de actividades sociales, disminución del afecto negativo y aumento de experiencias positivas (Antoni et al., 2006).

El análisis de la participación del estrés en el desarrollo de la enfermedad, es un tema que ha generado muchas investigaciones (Segerstrom \& Miller, 2004) ya que es parte del enfoque que la psicología ha tenido durante muchos años dirigido hacia los modelos patógenos de la salud y al abordaje de las debilidades del ser humano, teniendo una concepción de la persona que sufre alguna experiencia traumática como una víctima con potencial para desarrollar un trastorno (González, 2004).

Sin embargo, durante los últimos años ha crecido el interés en las distintas formas en que las personas hacen frente a las situaciones difíciles y estresantes que abordan en sus vidas (Chico, 2002); esto ha aumentando el interés en los recursos psicológicos que mejoran la salud, ya que estos funcionan como factores de protección psicológica relacionándose positivamente con la salud y el bienestar, y aumentan la resistencia a la adversidad facilitando una adecuada evaluación y afrontamiento de los cambios y dificultades de los sucesos vitales de la vida (Remor, Amorós \& Carrobles, 2006). 
Existen diferentes variables que se están estudiando debido a sus efectos positivos en la salud, entre las cuales se encuentran el apoyo social y el optimismo.

Según Cobb (1976) el apoyo social facilita enfrentar las crisis y la adaptación al cambio, además de protege al individuo en el paso de las transiciones y crisis del ciclo de la vida y de estados patológicos; también menciona que el apoyo social puede reducir la cantidad de medicación requerida y acelerar la recuperación y facilitar la adherencia al tratamiento.

Cohen y Wills (1985) propusieron dos modelos para explicar la asociación entre apoyo social y salud, uno es el modelo del efecto directo, el cual postula que el apoyo social favorece los niveles de salud, independiente de los niveles de estrés del individuo; y el otro es el modelo del efecto amortiguador, el cual postula que el apoyo social protege a los individuos de los efectos patógenos de los eventos estresantes; según Castro, Campero y Hernández (1997) estos modelos surgen de conceptualizar al apoyo social como variable antecedente o simultánea (modelo del efecto directo), o como una variable interviniente en la relación estrés enfermedad (modelo amortiguador).

El apoyo social se considera importante para el ajuste psicosocial ya que se ha encontrado que los grupos de apoyo social benefician el estado físico de las mujeres con cáncer de mama (Helgeson, Cohen, Schulz \& Yasko, 2000), además de incrementar la autoestima, el control percibido y significado de la experiencia (Helgeson \& Cohen, 1996).

Por otro lado, Turner-Cobb, Sephton, Koopman, Blake-Mortimer y Spiegel (2000) encontraron que un mayor apoyo social percibido disminuye la concentración de cortisol, mostrando la influencia del apoyo social sobre la disminución del estrés a través de un indicador fisiológico del mismo. Alferi, Carver, Antoni, Weiss y Durán (2001) señalan que el apoyo emocional e instrumental disminuye el distrés en mujeres con cáncer de mama después de una cirugía; asimismo, Trunzo y Pinto (2003) mencionan que el apoyo social afectivo media una asociación negativa entre el optimismo y el distrés.

En cuanto al optimismo, se ha relacionado con estrategias de afrontamiento más adaptativas (Carver, et al., 1993; Chang, 1998; Chico, 2002), ajuste psicológico y físico (Chang, 1998), menor frecuencia e intensidad de síntomas físicos (Remor, Amorós \& Carboles, 2006) y menor frecuencia de quejas somáticas (Martínez, Reyes, García \& González, 2006), además de asociarse con mayor supervivencia (Allison, Guichard, Fung \& Gilain, 2003).

Por otro lado, el estudio de Segerstrom, Taylor, Kemeny y Fahey (1998) sugiere que el optimismo se asocia con un menor grado de alteraciones en el humor y cambios en el sistema inmunológico durante situaciones estresantes, incrementando la función y el número de las células T. También se ha encontrado que las personas optimistas presentan menor estrés y depresión en situaciones estresantes, y cuentan con una red de apoyo social más amplia (Brissette, Scheier \& Carver, 2002).

En lo que se refiere a mujeres con cáncer de mama se ha encontrado que el optimismo predice niveles bajos de distrés (Carver et. al., 1993) y menor cantidad de síntomas de ansiedad y depresión (Epping-Jordan et al., 1999) además de estar relacionado con el ajuste a largo plazo (Carver, Smith, Antoni, Petronis, Weiss \& Derhagopian, 2005).

Carver, Lehman y Antoni (2003) mencionan que niveles bajos de optimismo en mujeres con cáncer de mama, se asocian a la disrupción social y de actividades recreativas, además de presentar mayor distrés y síntomas de fatiga.

El presente trabajo tiene como objetivo 1) describir las variables sociodemográficas de un grupo de mujeres con cáncer de mama, 2) describir los niveles y de la relación entre el estrés, el apoyo social, el optimismo y la salud en un grupo de mujeres con cáncer de mama y 3) Determinar los predictores (estrés, el apoyo social, el optimismo) de la salud en un de mujeres con cáncer de mama. 


\section{Método}

\section{Participantes}

Se utilizó una muestra no probabilística de mujeres diagnosticadas con cáncer de mama (n=72), sin embargo, el tamaño de la muestra se calculó con el Programa nQuery 6.0 (Gatsonis \& Sampson, 1989), para poder tener un mínimo aceptable de muestra para los análisis a realizar, con un nivel de significancia de .05 y un poder de 80, el tamaño estimado fue de 71 mujeres. Las cuales fueron entrevistadas en hospitales del área metropolitana de Monterrey, Nuevo León.

\section{Procedimiento}

La recolección de datos se llevó a cabo acudiendo a tres instituciones del área metropolitana de Monterrey, N. L., con previa autorización para la aplicación de los instrumentos; el instrumento fue autoaplicable y fue realizado en una sola sesión por las participantes, las condiciones de aplicación fueron diferentes dependiendo del espacio proporcionado en cada institución, sin embargo, las indicaciones e instrucciones fueron las mismas para cada participante, las cuales primeramente firmaban el consentimiento informado de la investigación (cubriendo el aspecto ético) y posteriormente contestaban el instrumento de evaluación.

Para el análisis estadístico de los datos se utilizó el programa SPSS 14.0, primeramente se realizaron los análisis descriptivos de cada variable, se calculó la consistencia interna de cada escala a través del alfa de Cronbach, posteriormente se analizaron las correlaciones entre las variables de estudio, utilizando el coeficiente de correlación de Pearson y por último, se llevo a cabo el análisis de regresión para evaluar las variables que explican la salud.

\section{Instrumentos}

Los cuestionarios utilizados en el estudio fueron los siguientes:

Escala de Estrés Percibido (Perceived stress scale, PSS). Fue elaborada por Cohen, Kamarak y Mermelstein (1983) para evaluar el grado en que las situaciones de la vida son valoradas como estresantes, obteniendo un alfa de Cronbach $(\alpha=.75)$. Se utilizó la adaptación cultural realizada en México por González y Landero (2007), la cual cuenta con 14 ítems y una consistencia interna $(\alpha=.83)$, además de confirmar la estructura factorial de la escala original, utilizando un análisis factorial confirmatorio. La puntuación va de nunca -0- a muy a menudo -4-. Invirtiéndose la puntuación en los ítems: 4, 5, 6, 7, 9, 10 y 13. La mayor puntuación corresponde a mayor estrés percibido.

Cuestionario de apoyo social funcional de Duke-UNC. Fue elaborado por Broadhead, Gehlbach, Degruy y Kaplan (1988) con el objetivo de medir “apoyo social funcional percibido”. Se utilizará la versión de Bellón, Delgado, Luna y Lardelli (1996), la cual consta de un índice de fiabilidad de .92 autoadministrada y .80 aplicada mediante entrevistador. Se trata de un cuestionario autoaplicado de 11 ítems, cada uno admite 5 posibles respuestas en una escala tipo Lickert. El análisis factorial original demuestra la existencia de dos dimensiones: apoyo confidencial (ítems 6, 7, 8, 9, y 10) y apoyo afectivo (ítems 4, 5 y 11). La puntuación obtenida con la suma de todos los ítems es un reflejo del apoyo percibido, no del real. A menor puntuación, menor apoyo.

Optimismo Life Orientation Test. Fue desarrollado por Scheier, Carver y Bridges (1994) consta de 10 ítems en escala Lickert de 5 puntos, 6 de los ítems miden el optimismo disposicional y los otros 4 sirven para hacer menos evidente el contenido del test; esta escala cuenta con una fiabilidad $(\alpha=$.79). Se utilizó la versión española de Otero, Luengo, Romero, Gómez y Castro (1998) del LOT-R. De los 6 ítems de optimismo disposicional, 3 están redactados en sentido positivo (1, 4, 10; optimismo) y 3 en sentido negativo (3, 7, 9; pesimismo). Las puntuaciones de los ítems redactados en sentido negativo se invierten y se obtiene una puntuación total orientada hacia el polo de optimismo, cuyo recorrido es de 6 a 30 .

Salud percibida. Se evaluó con ítem 29 de salud general, de la escala QLQ-30.3, elaborada por el grupo de Calidad de Vida de la Organización Europea para la Investigación y Tratamiento del 
Cáncer (EORTC). Este ítem evalúa la percepción de salud global durante la semana pasada a través de una escala que va de 1 (pésima salud) a 7 (excelente salud).

\section{Resultados}

\section{Descripción de la muestra}

Las características de la muestra se describen en la tabla 1, en la cual se puede observar que la edad media de las mujeres es de 53.5 años $(\mathrm{DE}=11.2)$, con una escolaridad promedio de 10.1 años $(\mathrm{DE}=5.1)$, y el promedio de hijos es de $2.7(\mathrm{DE}=1.7)$.

\section{TABLA 1}

Descripción de variables sociodemográficas ( $\mathrm{n}=72)$

\begin{tabular}{lccc}
\hline & Mediana & Media & D.E. \\
Edad & 55.0 & 53.5 & 11.2 \\
Escolaridad & 9.0 & 10.1 & 5.1 \\
Número de hijos & 2.0 & 2.7 & 1.7 \\
\hline
\end{tabular}

Se valoró la consistencia interna de las escalas utilizadas, encontrándose una adecuada consistencia interna, excepto la de optimismo en esta muestra. Los resultados de este análisis se presentan en la tabla 2.

TABLA 2

Consistencia interna de las escalas

\begin{tabular}{l|c}
\hline Escalas & $\alpha$ \\
Estrés & .79 \\
Apoyo social & .89 \\
Optimismo & .62 \\
\hline
\end{tabular}

Respecto a las variables psicosociales y la salud (tabla 3), la puntuación promedio del estrés es de 20.4 con una desviación típica de 6.4. En cuanto al apoyo social se obtuvo una puntuación promedio de 44.2 con una desviación típica de 7.9. Referente al optimismo la puntuación media es de 22.9 y la desviación típica es de 3.4; la puntuación promedio de salud es de 5.6 con una desviación típica de 1.2.

TABLA 3

Descripción de variables psicosociales y salud $(n=72)$

\begin{tabular}{l|c|c|c}
\hline & Mediana & Media & D.T. \\
Estrés & 20 & 20.4 & 6.4 \\
Apoyo Social & 45 & 44.2 & 7.9 \\
Optimismo & 22 & 22.9 & 3.4 \\
Salud & 6 & 5.6 & 1.2 \\
\hline
\end{tabular}


En cuanto al objetivo 2 se realizó el análisis de correlación entre las variables, el cual mostró las siguientes relaciones significativas: estrés y apoyo social $(\mathrm{r}=-.340, \mathrm{p}<.01)$; estrés y optimismo $(\mathrm{r}=$ -.383, $\mathrm{p}<.01)$; estrés y salud $(\mathrm{r}=-.499, \mathrm{p}<.01)$; optimismo y salud $(\mathrm{r}=.305, \mathrm{p}<.01)$, sin embargo, no se encontró relación significativa entre las siguientes variables: Apoyo social y optimismo $(r=.228$, $\mathrm{p}>$.05); y Apoyo social y salud ( $\mathrm{r}=.059, \mathrm{p}>.05)$.

Se procedió a realizar el análisis de regresión (tabla 4) para cumplir con el objetivo 3, para ello se utilizó el método por pasos con el criterio por default del programa (entrada de variable al .05 y .10 para salida de ellas del modelo). Los resultados fueron los siguientes, entraron solo dos variables, estrés en el primer modelo y, estrés y optimismo en el segundo modelo, el cambio de $\mathrm{F}$ fue significativo ( $\mathrm{p}<.05$ ), el valor del estadístico Durbin-Watson fue de 2.223, el índice de tolerancia fue de .964, por lo que se considera adecuado el modelo de regresión, explicando este el $22.3 \%$ de la varianza. Como se puede observar en la tabla 4, el coeficiente de determinación fue $.223\left(\mathrm{R}^{2}=.223\right)$, el coeficiente Beta de la variable estrés fue negativo y significativo $(B=.369 ; t=-3.418, p<.01)$ y el de optimismo fue positivo y significativo $(\mathrm{B}=.233$; $\mathrm{t}=2.155, \mathrm{p}<.05)$.

TABLA 4

Análisis de regresión

\begin{tabular}{|c|c|c|c|c|}
\hline Salud (VD) & $\mathbf{R}$ & B & Beta & $\mathbf{T}$ \\
\hline Constante & & 5.130 & & $4.696 * *$ \\
\hline Estrés percibido & $-.449 * *$ & -.070 & -.369 & $-3.418^{* *}$ \\
\hline Optimismo & $.295 *$ & .087 & .233 & $2.155^{*}$ \\
\hline
\end{tabular}

\section{Discusión}

El presente trabajo se planteó como objetivo describir las variables sociodemográficas, las variables psicosociales y la salud y la relación existente entre ellas, además de determinar las variables estudiadas que predicen la salud en un grupo de mujeres con cáncer de mama.

Los resultados obtenidos mostraron un nivel moderado de estrés y un nivel alto de apoyo social, optimismo y salud en las mujeres con cáncer de mama. Como se señaló en la introducción, la enfermedad de cáncer de mama es catalogada como estresante debido al impacto biopsicosocial que tiene su diagnóstico y tratamiento. Ante esta percepción, podríamos esperar un nivel alto de estrés en las mujeres con este padecimiento, sin embargo, los datos muestran un nivel moderado de estrés, que es importante tomar en cuenta debido a que la exposición constante a situaciones estresantes afecta la salud (Kirschbaum et al., 1995; Pereyra, 2006); por otro lado, una explicación para este nivel de estrés podrían ser las puntuaciones altas en apoyo social y optimismo, que son variables que funcionan como protectoras de la salud o moderadores del estrés (Cohen \& Wills, 1985; Chico, 2002; Brissette, Scheier \& Carver, 2002), y en este estudio se confirma la relación negativa del estrés con dichas variables, es decir a mayor apoyo social percibido y optimismo, menor nivel de estrés.

En cuanto al estrés y la salud se corroboró la relación negativa entre estrés y salud y se encontró una relación positiva entre optimismo y salud, estos hallazgos son similares a los de otros estudios realizados en muestras de estudiantes (Viñas \& Caparrós, 2000; Chang, 1998; Remor, et al., 2006), sin embargo, no se confirmó en esta muestra la relación directa entre apoyo social y salud, aunque es conocido que el apoyo social también actúa sobre la salud de manera indirecta moderando el estrés (Landero \& González, 2004), posiblemente esta condición también explique el hecho de que apoyo social no se encuentre como predictor. Las variables que se encontraron como predictores de salud percibida en esta muestra son estrés y el optimismo. 
Se considera que es importante seguir investigando acerca de las variables que tienen pueden estar afectando la salud de las mujeres con cáncer de mama ya sea positiva o negativamente, los resultados de este estudio nos indican la importancia de programas de intervención enfocados a reducir o controlar el estrés, e incluir en estos el objetivo de incrementar los pensamientos positivos.

Se sugiere para próximas investigaciones tomar en cuenta variables como el tiempo de diagnóstico, tipo y duración del tratamiento, así como otras variables que puedan explicar el comportamiento de este tipo de personas.

\section{Referencias}

Allison, P., Guichard, C., Fung, K., \& Gilain, L. (2003). Dispositional optimism predicts survival status 1 year after diagnosis in head and neck cancer patients. Journal of Clinical Oncology, 21(3), 543-548.

Alferi, S. Carver, C. Antoni, M. Weiss, S. \& Durán, R. (2001). An Exploratory Study of Social Support, Distress, and Life Disruption Among Low- Income Hispanic Women Under Treatment for Early Stage Breast Cancer. Healt Psychology, 20(1), 41- 46.

Antoni, M. Lechner, S. Kazi, A. Wimberly, S. Sifre, T. Urcuyo, K. Phillips, K. Glück, S. \& Carver, S. (2006). How Stress Management Improves Quality of Life After Treatment for Breast Cancer. Journal of Consulting and Clinical Psychology, 74(6), 1143-1152.

Arbizu, J. P. (2000). Factores psicológicos que interviene en el desarrollo del cáncer y la respuesta al tratamiento. ANALES Sis San Navarra, 24(1), 173-178.

Brissette, I., Scheier, M. \& Carver, C. (2002). The Role of Optimism in Social Network Development, Coping, and Psychological Adjustment During a Life Transition. Journal of Personality and Social Psychology, 82(1), 102-111.

Broadhead, W. Gehlbach, S. Degruy, F. Kaplan, B. (1988). The Duke-UNK functional social support questionnaire: measurement of social support in family medicine patients. Medical Care, 26, 709-23.

Bellón, J. Delgado, A. Luna, J. \& Lardelli, P. (1996). Validez y fiabilidad del cuestionario de apoyo social funcional Duke-UNC-11. Atención Primaria, 18, 153-63.

Cárdenas, J. \& Sandoval, F. (2006). Segunda Revisión del Consenso Nacional Sobre el Diagnóstico y Tratamiento del Cáncer Mamario. Revista Mexicana de Mastología, 1(1), 13-38.

Carver, C. Lehman, J. \& Antoni, M. (2003). Dispositional Pessimism Predicts Illness-Related Disruption of Social and Recreational Activities Among Breast Cancer Patients. Journal of Personality and Social Psychology, 84(4), 813-821.

Carver, C. Pozo, C. Harris, S. Noriega, V. Scheier, Robinson, D. Ketcham, A. Moffat, F. \& Clark, K. (1993). How coping mediates the effect of optimism on distress: A study of women with early stage breast cancer. Journal of Personality and social psychology, 65(2), 375- 390.

Carver, C., Smith, R., Antoni, M., Petronis, V., Weiss S. \& Derhagopian, R. (2005). Optimistic personality and psychosocial well-being during treatment predict psychosocial well-being among long-term survivors of breast cancer. Health Psychology, 24(5), 508-516.

Castro, R. Campero, L. \& Hernández, B. (1997). La investigación sobre apoyo social en salud: situación actual y nuevos desafíos. Journal of public health, 31(4), 425-435.

Cervera, S. \& Aubá, E. (2005). Calidad de vida y dinámica familiar tras diagnostico de cáncer de mama. Boletín de Psicología (85), 7-29. 
Chang, E. (1998). Dispositional Optimism and Primary and Secondary Appraisal of a Stressor: Controlling for Confounding Influences and Relations to Coping and Psychological and Physical Adjustment. Journal of Personality and Social Psychology, (74) 4, 1109-1120.

Chico, E. (2002). Optimismo disposicional como predictor de estrategias de afrontamiento. Psicothema, 14(3), 544- 550.

Cobb, S. (1976). Social support as a moderator of life stress. Psychosomatic Medicine, 38 (5), 300314.

Cohen, S. Kamarak, T. \& Mermelstein, R. (1983). A global measure of perceived stress. Journal of Health and Social Behaviour, 24, 385-396.

Cohen, S. \& Wills, T. (1985). Stress, social support, and the buffering hypothesis. Psychological Bulletin, 98 (2), 310-357.

Cruess, D. Antoni, M. Mcgregor, B. Kilbourn, K. Boyers, A. Alferi, S. Carver, C. \& Kumar, M. (2000). Cognitive-behavioral stress management reduces serum cortisol by enhancing benefit finding among women being treated for early stage breast cancer. Psychosomatic Medicine 62, 304-308.

Ehrenzweig, Y. (2006). Modelos de cognición social y adherencia terapéutica en pacientes con cáncer. En Oblitas, L. (Ed). (2006). Psicología de la Salud y Calidad de Vida. México: Thomson.

Epping-Jordan, J. Compas, B. Osowiecki, D. Oppedisano, G. Gerhardt, C. Primo, K. y Krag, D. (1999). Psychological adjustment in breast cancer: Processes of emotional distress. Health Psychology, 18(4), 315-326.

Gatsonis, C. \& Sampson, A.R. (1989). Multiple Correlation: Exact Power and Sample Size Calculations. Psychological Bulletin 106, 516-524.

Gómez, B. \& Escobar, A. (2002). La psiconeuroinmunología: bases de la relación entre los sistemas nervioso, endocrino e inmune. Revista de la Facultad de Medicina UNAM, 45(1), 22-26.

González, C. (2004). La psicología positiva: un cambio en nuestro enfoque patológico clásico. Liberabit, 10, 82-88.

González, M. y Landero, R. (2007). Factor Structure of the Perceived Stress Scale (PSS) in a Sample from Mexico. The Spanish Journal of Psychology, 10 (1), 199-206.

Helgeson, S. Cohen, S. Schulz, R. y Yasko, J. (2000). Group Support Interventions for Women With Breast Cancer: Who Benefits From What? Health Psychology, 19 (2), 107-114.

Helgeson, V. \& Cohen, S. (1996). Social Support and Adjustment to Cancer: Reconciling Descriptive, Correlational, and Intervention Research. Health Psychology, 15(2), 135-148.

Kirschbaum, C. Prussner, J. Stone, A. Federenko, L. Gaab, J. Lintz, D. Schommer, N. y Hellhammer, D. (1995). Persistent High Cortisol Responses to Repeated Psychological Stress in a Subpopulation of Healthy Men. Psychosomatic Medicine 57, 468-474.

Klinger, J., Herrera, J., Díaz, M., Jhann, A., Ávila, G. \& Tobar, C. (2005). La psiconeuroinmunología en el proceso salud enfermedad. Colombia Medica, 36(2), 120-129.

Landero, R. \& González, M-T. (2004). Variables psicosociales predictoras de las salud en las mujeres. Revista de Psicología Social, 19 (3), 255-264.

Martínez, A. Reyes, G. García, A. \& González, M. (2006). Optimismo/Pesimismo disposicional y estrategias de afrontamiento del estrés. Psicothema, 18(1), 66-72.

Otero, J.M., Luengo, A., Romero, E. Gómez, J.A. \& Castro, C. (1998). Psicología de personalidad. Manual de prácticas. Barcelona: Ariel Practicum. 
Pereyra, M. (2006). Estrés y Salud. En Oblitas, L. (Ed). (2006). Psicología de la Salud y Calidad de Vida. México: Thomson.

Phillips, K., Antoni, M., Lechner, S., Blomberg, B., Llabre, M., Avisar, E., Glück, S., Derhagopian, R. \& Carver, C. (2008). Stress management intervention reduces serum cortisol and increases relaxation during treatment for nonmetastatic breast cancer. Psychosomatic Medicine 70, 1-6.

Remor, E. Amorós, M \& Carrobles, J. (2006). Optimismo y la experiencia de la ira en relación con el malestar físico. Anales de psicología, 22(1), 37- 44.

Secretaría de Salud (2007). Epidemiología. Sistema Nacional de Vigilancia Epidemiológica, 24(31), $1-3$.

Segerstrom, S. \& Miller, G. (2004). Psychological stress and the human immune system: a metanalytic studi of 30 years of inquiry. Psychological Bulletin, 130(4), 601-630.

Segerstrom, S. Taylor, S. Kemeny, M. \& Fahey, J. (1998). Optimism Is Associated With Mood, Coping, and Immune Change in Response to Stress. Journal of Personality and Social Psychology, 74(6), 1646-1655.

Scheier, M., Carver, C. \& Bridges, M. (1994). Distinguishing optimism from neuroticism (and trait anxiety, self-mastery, and self-estem): A reevaluation of the Life Orientation Test. Journal of Personality and Social Psychology, 67, 1063-1078.

Sirera, R. Sánchez, P. \& Camps, C. (2006). Inmunología, Estrés, Depresión y Cáncer. Psicooncología, 3(1), 35-48.

Trunzo, J. \& Pinto, B. (2003). Social Support as a Mediator of Optimism and Distress in Breast Cancer Survivors. Journal of Consulting and Clinical Psychology, 71(4), 805-811.

Turner-Cobb, J. Sephton, S. Koopman, C. Blake-Mortimer, J. \& Spiegel, D. (2000). Social Support and Salivary Cortisol in Women With Metastatic Breast Cancer. Psychosomatic Medicine 62, 337-345.

Vinaccia, S. \& Orozco, L. (2005). Aspectos psicosociales asociados con la calidad de vida de personas con enfermedades crónicas. Diversitas, 1(2), 125-137.

Viñas, F. \& Caparrós, B. (2000). Afrontamiento del periodo de exámenes y sintomatología somática autoinformada en un grupo de estudiantes universitarios. Psicología.com. (revista electrónica), 4 (1). Revisado el 16 de septiembre del 2009. (Disponible en línea): http://www.psiquiatria.com/ psicologia/vol4num1/ art_5.htm.

Vera-Villarroel, P. \& Guerrero, A. (2003). Diferencias en habilidades en resolución de problemas sociales en sujetos optimistas y pesimistas. Universitas Psychologica, 2(1), 21-26.

Vera-Villarroel, P. \& Buela-Casal, G. (1999). Psiconeuroinmunología: Relaciones entre factores psicológicos e inmunitarios en humanos. Revista latinoamericana de Psicología, 31(2): 271289. 